\title{
SGLT2 inhibitors and lower limb complications: an updated meta-analysis
}

\author{
Chu Lin ${ }^{1 \dagger}$, Xingyun Zhu ${ }^{1 \dagger}$, Xiaoling Cai ${ }^{1 *}$, Wenjia Yang ${ }^{1}$, Fang LV ${ }^{1}$, Lin Nie ${ }^{2}$ and Linong $\mathrm{Ji}^{1 *}$
}

\begin{abstract}
Background: To exam the associations between the use of sodium glucose co-transporter 2 inhibitor (SGLT2i) and the risk of lower limb complications, and to analyze the associated factors.

Methods: Pubmed, Medline, Embase, the Cochrane Center Register of Controlled Trials for Studies and Clinicaltrial. gov were searched from the inception to November 2020. Randomized controlled trials of SGLT2i conducted in population containing diabetic patients with reports of amputation, peripheral arterial disease (PAD) and diabetic foot (DF) events were included. Random-effect model, fixed-effect model and meta-regression analysis were accordingly used.

Result: The numbers of SGLT2i users versus non-SGLT2i users in the analyses of amputation, PAD and DF were 40,925/33,414, 36,446/28,685 and 31,907/25,570 respectively. Compared with non-SGLT2i users, the risks of amputation and PAD were slightly increased in patients with canagliflozin treatment (amputation: $\mathrm{OR}=1.60,95 \% \mathrm{Cl} 1.04$ to 2.46; PAD: $\mathrm{OR}=1.53,95 \% \mathrm{Cl} 1.14$ to 2.05). Meta-regression analyses indicated that greater weight reduction in SGLT2i users was significantly associated with the increased risks of amputation $(\beta=-0.461,95 \% \mathrm{Cl}-0.726$ to -0.197$)$, $\operatorname{PAD}(\beta=-0.359,95 \% \mathrm{Cl}-0.545$ to -0.172$)$ and $\mathrm{DF}(\beta=-0.476,95 \% \mathrm{Cl}-0.836$ to -0.116$)$. Lower baseline diastolic blood pressure $(\beta=-0.528,95 \% \mathrm{Cl}-0.852$ to -0.205$)$, more systolic blood pressure reduction $(\beta=-0.207$, $95 \% \mathrm{Cl}-0.390$ to -0.023$)$ and more diastolic blood pressure reduction $(\beta=-0.312,95 \% \mathrm{Cl}-0.610$ to -0.015$)$ were significantly associated with the increased risks of amputation, PAD and DF respectively in patients with SGLT2i treatment.
\end{abstract}

Conclusions: The risks of amputation and PAD were slightly increased in patients with canagliflozin treatment. Reductions in body weight and blood pressure were associated with lower limb complications in patients with SGLT2i treatment.

Keywords: Sodium glucose co-transporter 2 inhibitor, Blood pressure lowering agent, Peripheral arterial disease, Amputation, Diabetic foot, Diabetes mellitus

\section{Background}

Sodium-glucose cotransporter 2 inhibitor (SGLT2i) is an effective hypoglycemic agent in the management of type 2 diabetes (T2D), with significant improvements in glycemic control and favorable cardiovascular/renal benefits

\footnotetext{
*Correspondence: dr_junel@sina.com; prof_jilinong@aliyun.com

${ }^{\dagger}$ Chu Lin and Xingyun Zhu contributed equally to this manuscript

${ }^{1}$ Department of Endocrinology and Metabolism, Peking University People's Hospital, Beijing, China

Full list of author information is available at the end of the article
}

[1]. However, since almost two-fold incidence of amputation was reported in patients with canagliflozin treatment in CANVAS program, the United States Food and Drug Administration (FDA) issued a Boxed Warning to canagliflozin drug label $[2,3]$. Although the Boxed Warning to the risk of amputation with canagliflozin has been encouragingly removed by FDA in the late August 2020, such unexpected concern still cast a shade on SGLT2i treatment in patients at high risk of lower limb complications [4]. As FDA suggested, despite the risk of amputation might be lower than previously described, a close 
monitoring is still needed to guarantee the safe application of canagliflozin [4].

Recently, several systematic reviews and meta-analyses have re-evaluated the association between SGLT2i and amputation, which indicated that SGLT2i was not associated with the increased risk of amputation [5, 6]. However, does it mean that we are in no worries to embrace the promoted applications of SGLT2i? It is noted that even though the Box Warning has been removed, the potential risk of major amputation with canagliflozin in patients with advanced atherosclerosis and impaired peripheral circulation still exists [4]. A large cohort study by using US database claimed that the increase in the rate of amputation with canagliflozin was most apparent among adults aged 65 or older with baseline cardiovascular disease [7]. As is known, the majority of lower limb amputation in patients with diabetes was due to uncontrolled infection and subsequent necrosis of extremities, which may be associated with other relevant conditions including peripheral arterial disease (PAD) and diabetic foot (DF) [8]. Such conditions may precede the surgical procedures and eventually lead to amputation. So far, the evaluations of PAD and DF are less characterized than amputation in patients with SGLT2i treatment.

Moreover, the explanations for the increased risk of amputation observed in patients with canagliflozin treatment are not definite. One hypothesis emphasized the hemodynamic effects of diuresis and blood pressure reduction within canagliflozin treatment. Similar effects were also found in diuretics, which might worsen the peripheral perfusion and in turn increase the risk of lower limb amputation in patients with T2D [9]. However, such scientific assumption has not been fully tested experimentally and clinically.

Therefore, we designed and performed an updated meta-analysis using data from randomized controlled trials (RCTs) of SGLT2i conducted in population containing diabetic patients, to hopefully produce a comprehensive safety evaluation on amputation, PAD and DF in patients using SGLT2i, and more importantly, to assess the association between altered hemodynamic status (diuresis and blood pressure reduction) and the risk of lower limb complications in SGLT2i users. Our study aims to provide state-of-the-art evidence-based guidance for appropriate clinical application of SGLT2i especially in patients at high risk of lower limb complications.

\section{Methods}

\section{Data sources and searches}

According to recommendations from the Cochrane Handbook for Systematic Reviews for meta-analysis, two independent investigators (NL and XZ) conducted systematic searches respectively for RCTs of SGLT2i in
Pubmed, Medline, Embase, the Cochrane Center Register of Controlled Trials for Studies and Clinicaltrial.gov from the inception to November 2020. The search terms were as follows: type 1 diabetes (T1D); T2D; SGLT2i; cardiovascular outcome trial (CVOT); renal outcome trial (ROT); efficacy; safety; placebo controlled; RCT. The search strategies were as follows: (1) "T1D or T2D" and "CVOT or ROT" and "SGLT2i"; (2) "T1D or T2D" and "efficacy or safety" and "SGLT2i" and "RCT"; (3) "T1D or T2D" and "placebo controlled" and "SGLT2i" and "RCT". The searching results from two investigators were checked together to form the final database for study selection. Reference lists of relevant articles were also screened in order not to miss any possibly eligible study.

\section{Study selection}

The inclusion criteria for eligible studies were as follows: (1) RCTs conducted in population containing diabetic patients; (2) RCTs with reports of PAD, amputation and DF events; (3) RCTs with SGLT2i and placebo or nonSGLT2i active control in different treatment arms. The exclusion criteria for the meta-analysis for SGLT2i treatment were as follows: (1) RCTs without diabetic patients at all; (2) RCTs without reports of PAD, amputation and DF event.

\section{Data extraction and quality assessment}

Two investigators (NL and XZ) abstracted data of all studies, including the publication infomation, study design, treatment arms, study duration, baseline characteristics, efficacy endpoints such as HbA1c change, weight change and blood pressure change etc., lower limb complication event, and assessed the qualities of the included studies. Another two investigators (CL and WY) checked the abstractions and assessments for accuracy. The risk of bias was evaluated using the Cochrane risk of bias tool. PAD, amputation and DF events would be extracted from clinicaltrial.gov website with unique registered RCT number if the data was absent in both articles and supplementary materials. PAD is defined as the narrowing or blockage of the vessels that carry blood from the heart to the legs. The pre-specific PAD events included in this meta-analysis were as follows: arterial thrombosis limb, arterial stenosis limb, ischemic limb pain, iliac artery stenosis, iliac artery occlusion, iliac artery embolism, femoral artery occlusion, femoral arterial stenosis, extremity necrosis, intermittent claudication, peripheral arterial occlusive disease, peripheral artery occlusion, peripheral artery ischemia, peripheral artery stenosis, peripheral artery thrombosis, peripheral artery circulatory failure and leg ischemia. We addressed discrepancies by 
inviting a third investigator (FL) to join the discussion, and resolved disagreements by consensus.

\section{Data synthesis and analysis}

Continuous outcomes were evaluated by computing the weighted mean differences (WMDs) and 95\% confidence intervals (CIs). Categorical outcomes were evaluated by computing the odd ratios (ORs) and accompanying 95\% CIs. Higgins $\mathrm{I}^{2}$ statistics were used to evaluate the between-study heterogeneity, when an $\mathrm{I}^{2}$ value $>50 \%$ indicating high level of heterogeneity. Fixed-effects model was used for low level of heterogeneity and random-effects model was used for high level of heterogeneity. Meta-regression analyses were performed to evaluate the associations between different potential associated factors and the risks of PAD, amputation and DF. Publication bias was assessed via funnel plot tests. Statistical significance was considered at $\mathrm{P}<0.05$.

Statistical analyses were primarily performed by using the STATA statistical software package (version 11.0, Stata Corp, College Station, Texas, USA) and the Review Manager statistical software package (version 5.3, Nordic Cochrane Centre, Copenhagen, Denmark). Analyses were conducted according to the Preferred Reporting Items for Systematic Reviews and Meta-Analyses (PRISMA) guidelines for conducting and reporting meta-analyses of RCTs.

\section{Results}

\section{Included studies}

In total, there were 39 RCTs of SGLT2i treatment included in our study, among which 31 RCTs, 15 RCTs and 19 RCTs were included in the analyses for PAD, amputation and DF respectively (Additional file 1: Figure S1). The numbers of SGLT2i users versus non-SGLT2i users in the analyses of amputation, PAD and DF were $40,925 / 33,414,36,446 / 28,685$ and $31,907 / 25,570$ respectively. The majority of enrolled RCTs with SGLT2i treatment were conducted in patients with diabetes, with 33 RCTs in T2D, 4 RCTs in T1D and 3 RCTs in mixed population of diabetic and non-diabetic patients. The baseline characteristics of all included RCTs were systematically summarized in Additional file 1: Table S1.

The risk of bias was evaluated by the Cochrane instrument, which suggested the overall risk of bias and the selective reporting was low (Additional file 1: Table S2). The publication bias was accessed by funnel plot test, which all showed even distributions (Additional file 1: Figures S2-S4).

\section{Risk of amputation, PAD and DF in patients with SGLT2i treatment}

Compared with non-SGLT2i users, the risk of amputation $(\mathrm{OR}=1.23,95 \% \mathrm{CI} 1.08$ to $1.40, \mathrm{P}=0.002$ ) (Fig. 1a) and the risk of PAD (OR $=1.21,95 \%$ CI 1.03 to 1.42 , $\mathrm{P}=0.02$ ) (Fig. $1 \mathrm{~b}$ ) were slightly increased in patients with SGLT2i treatment, which were mainly driven by the results from CVOTs and ROTs (Table 1). The drug-type analysis indicated that the increased risk of amputation $(\mathrm{OR}=1.60,95 \% \mathrm{CI} 1.04$ to $2.46, \mathrm{P}=0.03)$ and the risk of $\operatorname{PAD}(\mathrm{OR}=1.53,95 \% \mathrm{CI} 1.14$ to $2.05, \mathrm{P}=0.005)$ were only observed in canagliflozin subgroup but not in other subtypes of SGLT2i. As for study population, the incidence of amputation $(\mathrm{OR}=1.24,95 \% \mathrm{CI} 1.08$ to $1.42, \mathrm{P}=0.002)$ and PAD $(\mathrm{OR}=1.23,95 \% \mathrm{CI} 1.03$ to $1.45, \mathrm{P}=0.02)$ were significantly increased in SGLT2i users versus nonSGLT2i users, which was only observed in pure diabetic population strata (Table 1). Moreover, the risk of amputation $(\mathrm{OR}=1.22,95 \% \mathrm{CI} 1.07$ to $1.39, \mathrm{P}=0.02)$ and the risk of PAD $(\mathrm{OR}=1.22,95 \% \mathrm{CI} 1.03$ to $1.44, \mathrm{P}=0.02)$ were significantly higher in RCTs with study duration longer than 52 weeks, when compared with non-SGLT2i users (Table 1). However, the incidence of DF was not elevated in SGLT2i users, which remained quite consistent among multiple sensitivity analyses (Table 1).

\section{Associated factors with the risk of amputation, PAD and DF in SGLT2i treatment}

According to the results of meta-regression analyses, greater weight reduction in SGLT2i users was significantly associated with the risk of amputation $(\beta=$ $-0.461,95 \% \mathrm{CI}-0.726$ to $-0.197, \mathrm{P}=0.007)$, the risk of PAD $(\beta=-0.359,95 \% \mathrm{CI}-0.545$ to $-0.172, \mathrm{P}<0.001)$ and the risk of DF $(\beta=-0.476,95 \% \mathrm{CI}-0.836$ to $-0.116, \mathrm{P}=0.012$ ) (Fig. 2; Tables 2, 3 and 4). Consistently, greater weight reduction difference between SGLT2i and control groups was also significantly associated with the risk of amputation $(\beta=-0.436,95 \% \mathrm{CI}-0.795$ to $-0.078, \mathrm{P}=0.026)$, the risk of PAD $(\beta=-0.264,95 \%$ $\mathrm{CI}-0.472$ to $-0.056, \mathrm{P}=0.014)$ and the risk of $\mathrm{DF}(\beta$ $=-0.437,95 \% \mathrm{CI}-0.719$ to $-0.155, \mathrm{P}=0.004)$ in SGLT2i users (Fig. 2; Tables 2, 3 and 4). Moreover, lower baseline diastolic blood pressure $(\beta=-0.528,95 \% \mathrm{CI}$ -0.852 to $-0.205, P=0.009)$, more systolic blood pressure reduction $(\beta=-0.207,95 \% \mathrm{CI}-0.390$ to -0.023 , $\mathrm{P}=0.028)$ and more diastolic blood pressure reduction $(\beta$ $=-0.312,95 \% \mathrm{CI}-0.610$ to $-0.015, \mathrm{P}=0.041$ ) were significantly associated with increased risks of amputation, PAD and DF respectively in SGLT2i users (Fig. 2; Tables 2, 3 and 4). Other factors such as age, sex, body mass index, duration of diabetes, renal function, previous history of PAD, or HbA1c reduction achieved by SGLT2i, 


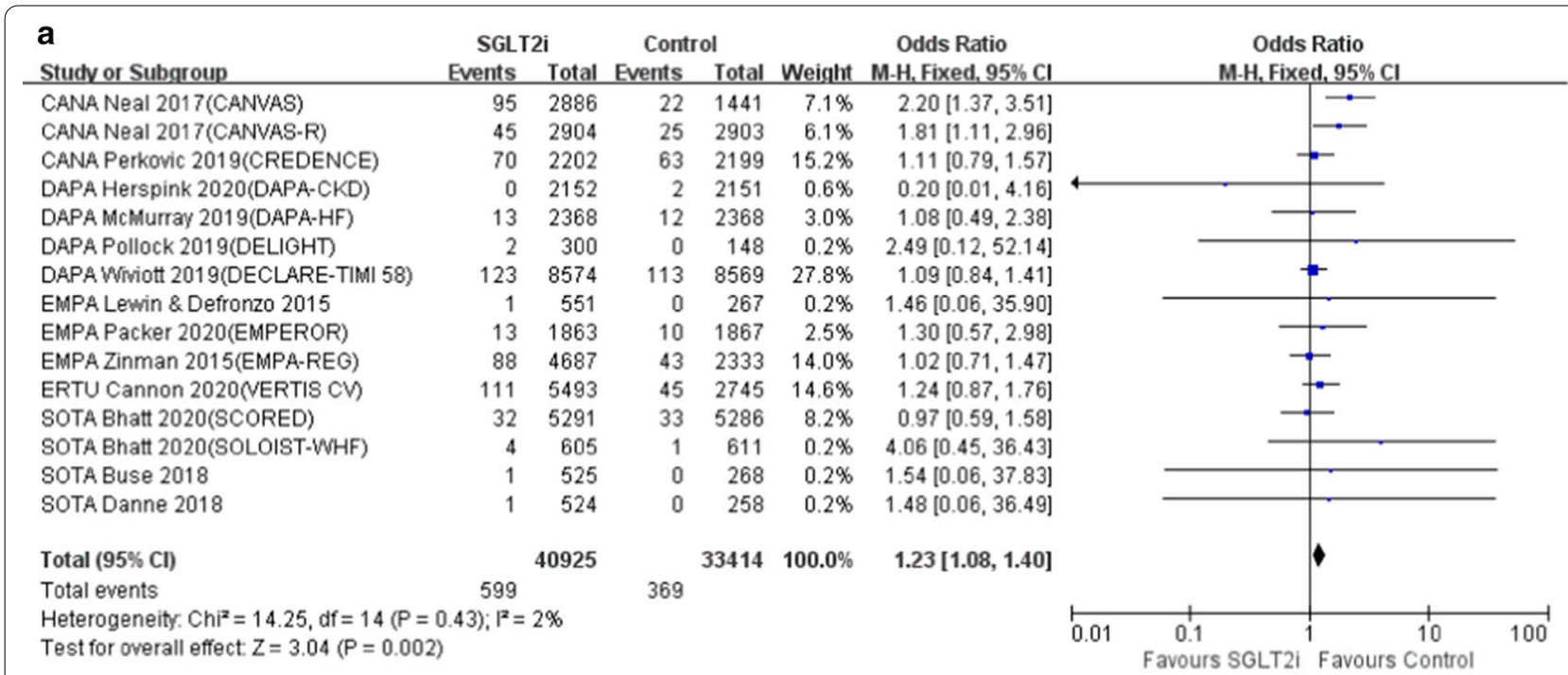

\section{b}

SGLT2i Control Odds Ratio

Studv or Subgroup

Events Total Events Total Weight M-H, Fixed, $95 \% \mathrm{CI}$

CANA Bode 2015

CANA Leiter 2015

477

CANA Neal 2017(CANVAS)

CANA Neal 2017 (CANVAS-R)

CANA Perkovic 2019(CREDENCE)

CANA Rosenstock 2016

CANA Schemthaner 2013

CANA Yale 2014

DAPA Dandona 2017(DEPICT-1)

DAPA Ferrannini 2010

DAPA Handelsman 2018

DAPA. Herspink 2020(DAPA-CKD)

DAPA Leiter 2014

DAPA Matthaei 2015

DAPA McMurray 2019(DAPA-HF)

DAPA Müller-Wieland 2018

DAPA Schumm-Draeger 2014

DAPA Wiviott 2019(DECLARE-TIMI 58)

EMPA Barnett 2014

EMPA Hadjadj 2016

EMPA Haring 2013

EMPA Haring 2014

EMPA Kovas 2014

EMPA Ridderstrăle 2014

EMPA Rosenstock 2014

EMPA Rosenstock 2015

EMPA Zinman 2015(EMPA-REG)

ERTU Grunberger 2017

ERTU Hollander 2019

ERTU Rosenstock 2018

SOTA Grag 2017

$\begin{array}{rr}1 & 477 \\ 1 & 968 \\ 74 & 2886 \\ 36 & 2904 \\ 25 & 2202 \\ 0 & 474 \\ 1 & 377 \\ 1 & 179 \\ 1 & 518 \\ 1 & 410 \\ 1 & 232 \\ 20 & 2152 \\ 2 & 480 \\ 0 & 109 \\ 14 & 2368 \\ 1 & 626 \\ 1 & 199 \\ 102 & 8574 \\ 1 & 321 \\ 1 & 326 \\ 1 & 441 \\ 1 & 430 \\ 0 & 330 \\ 0 & 765 \\ 2 & 375 \\ 1 & 324 \\ 99 & 4687 \\ 2 & 313 \\ 1 & 888 \\ 2 & 412 \\ 0 & 699\end{array}$

0 237

$\begin{array}{llll}0 & 482 & 0.20 & 1.50[0.06,36.84]\end{array}$

$\begin{array}{ll}19 & 1441\end{array}$

232903

232199

1237

$\begin{array}{ll}0 & 378\end{array}$

$0 \quad 90$

0260

$\begin{array}{rr}0 & 260 \\ 0 & 75\end{array}$

0 229

$18 \quad 2151$

$\begin{array}{ll}3 & 482\end{array}$

1109

$13 \quad 2368$

$\begin{array}{lr}1 & 313\end{array}$

$0 \quad 101$

$100 \quad 8569$

$0 \quad 319$

$0 \quad 168$

$0 \quad 225$

0207

1168

2780

$\begin{array}{ll}0 & 188\end{array}$

$0 \quad 170$

$38 \quad 2333$

$\begin{array}{ll}1 & 154\end{array}$

0 437

0 209

1703

36446

Total $(95 \% \mathrm{Cl})$

393

Total events

Heterogeneity. $C \mathrm{Ch}^{2}=14.52, \mathrm{df}=30(\mathrm{P}=0.99) ; \mathrm{I}^{2}=0 \%$ 245

$28685100.0 \%$

$9.0 \%$

$8.3 \%$

$8.3 \%$

$0.7 \%$

$0.2 \%$

$0.2 \%$

$0.2 \%$

$0.3 \%$

$0.2 \%$
$6.5 \%$

$6.5 \%$
$1.1 \%$

$0.5 \%$

$4.7 \%$

$0.5 \%$

$0.2 \%$

$36.0 \%$

$0.2 \%$

$0.2 \%$

$0.2 \%$

$0.2 \%$

$0.9 \%$

$0.2 \%$
$0.2 \%$

$18.1 \%$

$0.5 \%$

$0.2 \%$

$0.2 \%$

$0.5 \%$

$1.97[1.18,3.27]$

$1.57[0.93,2.66]$

$1.09[0.61,1.92]$

$0.17[0.01,4.09]$

$3.02[0.12,74.27]$

$1.52[0.06,37.71]$

$1.51[0.06,37.20]$

$0.55[0.02,13.71]$

$2.97[0.12,73.39]$

$1.11[0.59,2.11]$

$0.67[0.11,4.02]$

$0.33[0.01,8.20]$

$1.08[0.51,2.30]$

$0.50[0.03,8.01]$

$1.53[0.06,37.99]$

$1.02[0.77,1.35]$

$2.99[0.12,73.69]$

$1.55[0.06,38.33]$

$1.54[0.06,37.85]$

$1.45[0.06,35.73]$

$0.17[0.01,4.17]$

$0.20[0.01,4.24]$

$2.52[0.12,52.83]$

$1.58[0.06,39.02]$

$1.30[0.89,1.90]$

$0.98[0.09,10.94]$

$1.48[0.06,36.38]$

$2.55[0.12,53.39]$

$0.33[0.01,8.23]$

$1.21[1.03,1.42]$

Test for overall effect: $Z=2.34(P=0.02)$

Fig. 1 The risk of amputation and PAD in patients with SGLT2i treatment. a The risk of amputation in patients with SGLT2i treatment. b The risk of PAD in patients with SGLT2i treatment. PAD, peripheral arterial disease; SGLT2i, sodium glucose co-transporter 2 inhibitor
Odds Ratio

M.H. Fixed, $95 \% \mathrm{Cl}$
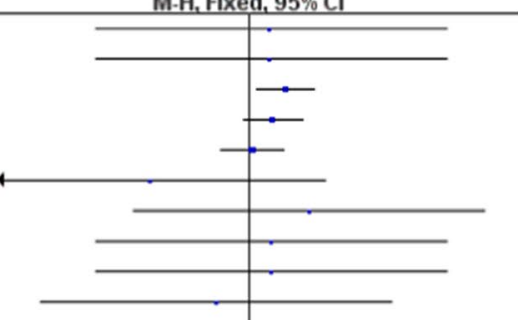

4

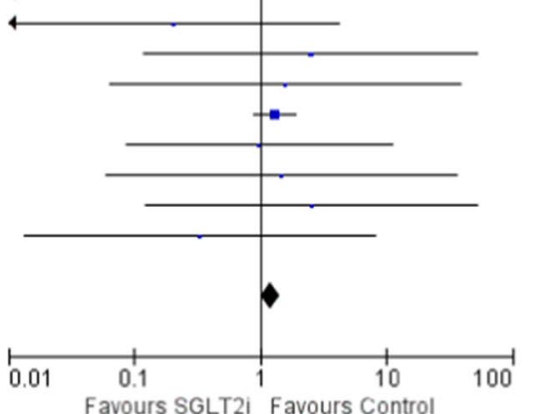


Table 1 Risk of amputation, PAD and DF events in patients with SGLT2i treatment

\begin{tabular}{|c|c|c|c|c|c|}
\hline Subgroup & $\begin{array}{l}\text { No. of participants } \\
\text { (SGLT2i/control) }\end{array}$ & OR & $95 \% \mathrm{Cl}$ & P value & $I^{2}(\%)$ \\
\hline \multicolumn{6}{|c|}{ Risk of amputation by SGLT2i subtypes } \\
\hline In total* & $40,925 / 33,414$ & 1.23 & $1.08,1.40$ & 0.002 & 2 \\
\hline Canagliflozin* & $7992 / 6543$ & 1.60 & $1.04,2.46$ & 0.03 & 67 \\
\hline Dapagliflozin & $13,394 / 13,236$ & 1.08 & $0.85,1.37$ & 0.54 & 0 \\
\hline Empagliflozin & $7101 / 4467$ & 1.07 & $0.76,1.49$ & 0.71 & 0 \\
\hline Ertugliflozin & $5493 / 2745$ & 1.24 & $0.87,1.76$ & 0.23 & NA \\
\hline Sotagliflozin & $6945 / 6423$ & 1.08 & $0.68,1.70$ & 0.75 & 0 \\
\hline \multicolumn{6}{|l|}{ Risk of amputation by study types } \\
\hline CVOT and ROT* & $39,025 / 32,473$ & 1.23 & $1.07,1.40$ & 0.003 & 29 \\
\hline Efficacy and safety evaluation & 1900/941 & 1.74 & $0.36,8.39$ & 0.49 & 0 \\
\hline \multicolumn{6}{|l|}{ Risk of amputation by population } \\
\hline DM only* & $34,872 / 27,196$ & 1.24 & $1.08,1.42$ & 0.002 & 14 \\
\hline Including patients without DM & $6383 / 6386$ & 1.08 & $0.62,1.88$ & 0.78 & 0 \\
\hline \multicolumn{6}{|l|}{ Risk of amputation by control types } \\
\hline Active agent & $551 / 267$ & 1.46 & $0.06,35.90$ & 0.82 & NA \\
\hline Placebo* & $40,374 / 33,147$ & 1.23 & $1.08,1.40$ & 0.002 & 9 \\
\hline \multicolumn{6}{|c|}{ Risk of amputation by study duration } \\
\hline$<26$ weeks & $300 / 148$ & 2.49 & $0.12,52.14$ & 0.56 & 0 \\
\hline 26-52 weeks & $2205 / 1404$ & 2.34 & $0.58,9.52$ & 0.23 & 0 \\
\hline$>52$ weeks $^{*}$ & $33,129 / 26,576$ & 1.24 & $1.08,1.43$ & 0.002 & 33 \\
\hline \multicolumn{6}{|l|}{ Risk of PAD by SGLT2i subtypes } \\
\hline In total $\left.\right|^{*}$ & $36,446 / 28,685$ & 1.21 & $1.03,1.42$ & 0.02 & 0 \\
\hline Canagliflozin* & $10,467 / 7967$ & 1.53 & $1.14,2.05$ & 0.005 & 0 \\
\hline Dapagliflozin & $15,668 / 14,657$ & 1.03 & $0.81,1.30$ & 0.83 & 0 \\
\hline Empagliflozin & $7999 / 4558$ & 1.26 & $0.88,1.78$ & 0.20 & 0 \\
\hline Ertugliflozin & $1613 / 800$ & 1.50 & $0.30,7.43$ & 0.62 & 0 \\
\hline Sotagliflozin & $699 / 703$ & 0.33 & $0.01,8.23$ & 0.50 & NA \\
\hline \multicolumn{6}{|l|}{ Risk of PAD by study types } \\
\hline CVOT and $\mathrm{ROT}^{*}$ & $25,773 / 21,964$ & 1.24 & $1.05,1.46$ & 0.01 & 6 \\
\hline Efficacy and safety evaluation & $10,673 / 6721$ & 0.96 & $0.55,1.68$ & 0.90 & 0 \\
\hline \multicolumn{6}{|l|}{ Risk of PAD by population } \\
\hline DM only* & $31,926 / 24,166$ & 1.23 & $1.03,1.45$ & 0.02 & 0 \\
\hline Including patients without DM & $4520 / 4519$ & 1.10 & $0.67,1.79$ & 0.71 & 0 \\
\hline \multicolumn{6}{|l|}{ Risk of PAD by control types } \\
\hline Active agent & $3856 / 2619$ & 1.00 & $0.33,3.06$ & 1.00 & 0 \\
\hline Placebo* & $32,590 / 26,066$ & 1.22 & $1.03,1.43$ & 0.02 & 0 \\
\hline \multicolumn{6}{|l|}{ Risk of PAD by study duration } \\
\hline$<26$ weeks & $5043 / 3159$ & 0.91 & $0.43,1.90$ & 0.80 & 0 \\
\hline 26-52 weeks & $2766 / 1725$ & 1.70 & $0.50,5.76$ & 0.40 & 0 \\
\hline$>52$ weeks* $^{*}$ & $28,637 / 23,801$ & 1.22 & $1.03,1.44$ & 0.02 & 0 \\
\hline \multicolumn{6}{|l|}{ Risk of DF by SGLT2i subtypes } \\
\hline In total & $31,907 / 25,570$ & 1.24 & $0.93,1.64$ & 0.14 & 0 \\
\hline Canagliflozin & $9139 / 7115$ & 1.55 & $0.94,2.54$ & 0.09 & 0 \\
\hline Dapagliflozin & $14,638 / 13,809$ & 1.20 & $0.79,1.83$ & 0.40 & 0 \\
\hline Empagliflozin & $7029 / 4055$ & 0.90 & $0.48,1.67$ & 0.74 & 0 \\
\hline Ertugliflozin & $1201 / 591$ & 1.48 & $0.15,14.28$ & 0.73 & 0 \\
\hline \multicolumn{6}{|l|}{ Risk of DF by study types } \\
\hline CVOT and ROT & $25,773 / 21,964$ & 1.23 & $0.91,1.66$ & 0.17 & 0 \\
\hline Efficacy and safety evaluation & $6134 / 3606$ & 1.26 & $0.55,2.92$ & 0.59 & 0 \\
\hline
\end{tabular}


Table 1 (continued)

\begin{tabular}{|c|c|c|c|c|c|}
\hline Subgroup & $\begin{array}{l}\text { No. of participants } \\
\text { (SGLT2i/control) }\end{array}$ & OR & $95 \% \mathrm{Cl}$ & $P$ value & $I^{2}(\%)$ \\
\hline \multicolumn{6}{|l|}{ Risk of DF by population } \\
\hline DM only & $27,387 / 21,051$ & 1.28 & $0.95,1.72$ & 0.11 & 0 \\
\hline Including patients without DM & $4520 / 4519$ & 0.89 & $0.34,2.31$ & 0.81 & 0 \\
\hline \multicolumn{6}{|l|}{ Risk of DF by control types } \\
\hline Active agent & $4173 / 2467$ & 1.54 & $0.44,5.34$ & 0.50 & 0 \\
\hline Placebo & $27,734 / 23,103$ & 1.22 & $0.91,1.63$ & 0.18 & 0 \\
\hline \multicolumn{6}{|l|}{ Risk of DF by study duration } \\
\hline$<26$ weeks & $1131 / 562$ & 1.49 & $0.23,9.51$ & 0.67 & 0 \\
\hline $26-52$ weeks & $2940 / 1614$ & 1.51 & $0.44,5.16$ & 0.51 & 0 \\
\hline$>52$ weeks & $27,836 / 23,394$ & 1.22 & $0.91,1.63$ & 0.19 & 0 \\
\hline
\end{tabular}

${ }^{*} \mathrm{P}<0.05$

$P A D$ peripheral arterial disease, SGLT2i sodium glucose co-transporter 2 inhibitor, DF diabetic foot, DM diabetes mellitus, CVOT cardiovascular outcome trial, $R O T$ renal outcome trial, OR odd ratio, Cl confidence interval, NA not applicable

were not associated with the risk of lower limb complications (Tables 2, 3 and 4).

\section{Discussion}

By using the data from RCTs of SGLT2i, we found that the risks of amputation and PAD were slightly increased in patients using SGLT2i, mainly derived from the use of canagliflozin. But the use of SGLT2i did not increase the incidence of DF events. Furthermore, we found that the body weight reduction and blood pressure reduction in SGLT2i users were associated with the risks of amputation, PAD and DF, which might provide new insights and supporting evidence for the hemodynamic hypothesis and promote the evidence-based prevention of lower limb complications concerning the application of SGLT2i in the future. In addition to routine foot care, monitoring body weight and blood pressure change might be a useful precaution action for risk management during the SGLT2i treatment course, especially for patients at high risk of lower limb complications.

SGLT2i reduces plasma glucose by increasing the renal threshold for glucose reabsorption, leading to increased urinary glucose excretion and osmotic diuresis, which may be associated with a reduction in intravascular volume [10]. According to the results from this meta-analysis, we supposed that the reductions in body weight and blood pressure might indirectly reflect the body fluid loss and altered hemodynamic status in SGLT2i users. Reductions in body weight by SGLT2i are mainly mediated by two mechanisms: osmotic diuresis and urinary glucose excretion. Previous studies indicated that the early phase of weight reduction in SGLT2i users was caused by the enhanced diuresis and the increased loss of intravascular volume, since the adiposity volume was mildly changed up to first 4-8 weeks [11-13]. When excess body fluid was excreted and a dry body weight was reached, SGLT2i continued to reduce or maintain the body weight by urinary glucose excretion and additional regulatory effects on metabolism [14], which might play greater role in the later phase of weight reduction. Actually, an increased risk of lower extremity amputation was previously reported in patients with T2D using thiazide diuretics [9]. Diuretic-induced volume depletion might contribute to the circulatory failure in the distal peripheral arterial beds and consequently worsened the insufficient perfusion in the extremities. Therefore, similar diuretic effect of SGLT2i may also be associated with insufficient perfusion, which subsequently facilitates the development of lower limb complications in those susceptible patients.

Whether the weight reduction achieved through urinary glucose excretion with SGLT2i treatment plays a role in the risk of lower limb complications? To answer this question, another anti-diabetic agent, glucagonlike peptide-1 receptor analog (GLP-1RA), might give us the hints. GLP-1RA, enhances glucose-dependent insulin secretion, inhibits glucagon secretion, slows gastric emptying, and reduces food intake $[15,16]$. Unlike SGLT2i, GLP-1RA leads to weight reduction mainly through energy intake restriction without diuretic effects. According to our previous meta-analysis of 97 RCTs, we proved that treatment of SGLT2i and GLP-1RA led to comparable weight changes from baseline, which were both with significance when compared with placebo treatment [17]. However, no reports of the increased risk of amputation events in patients with GLP-1RA treatment were observed, which is consistent with the observations in the real-world study [18]. Moreover, a nationwide register-based cohort study indicated that 


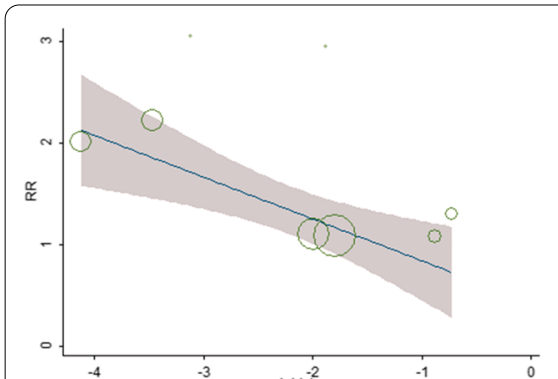

(a)

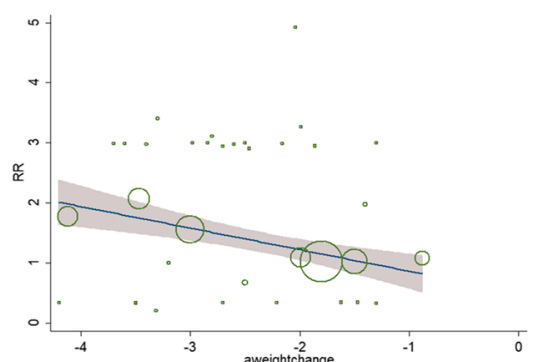

(d)

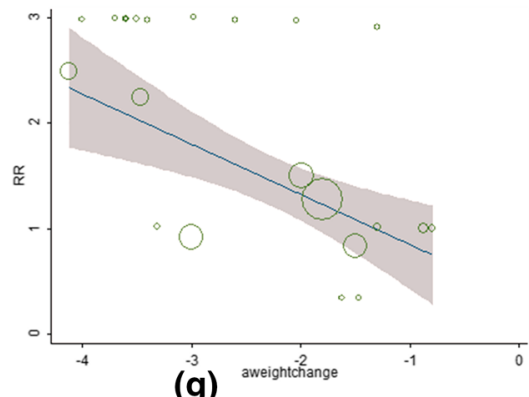

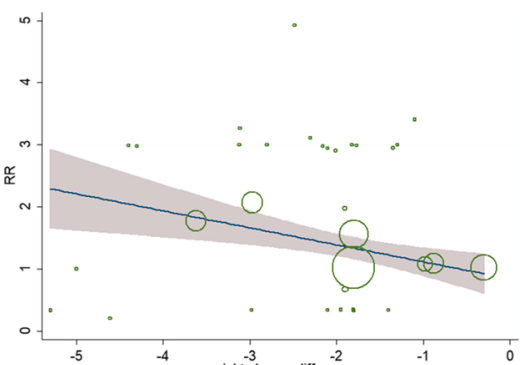

(b)

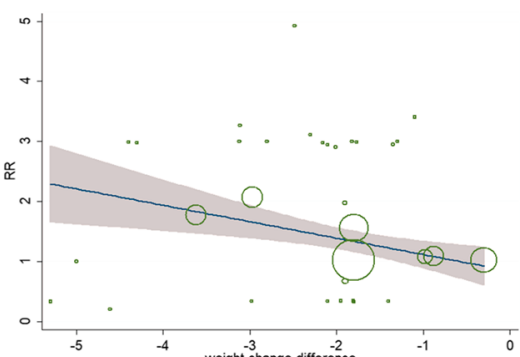

(e)

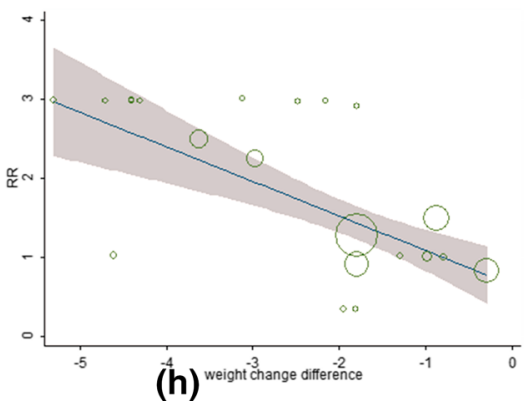

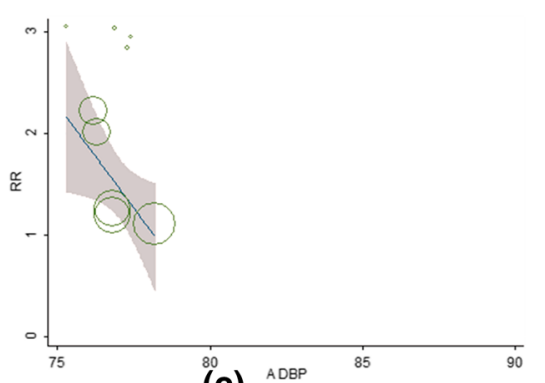

(c)

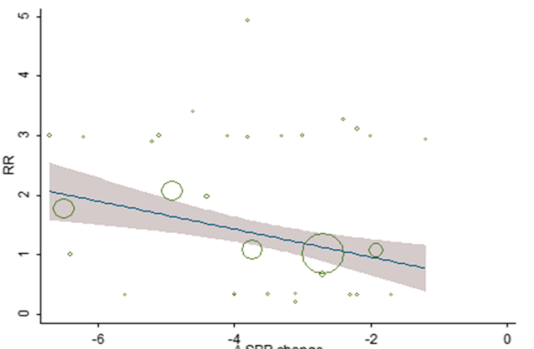

(f)

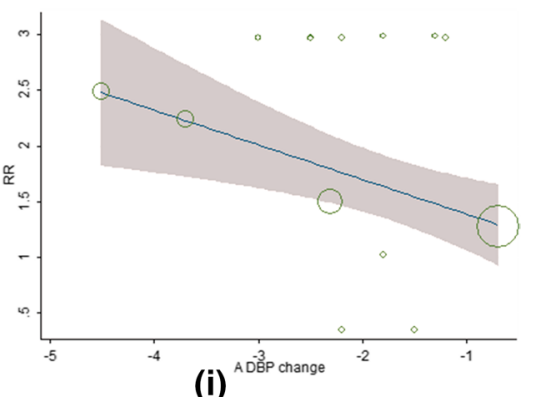

(i)

Fig. 2 Meta-regression analysis for the associated factors and risk of lower limb complications in patients with SGLT2i treatment. a The association between weigh change and risk of amputation in patients with SGLT2i treatment. $\mathbf{b}$ The association between weigh change difference and risk of amputation in patients with SGLT2i treatment. c The association between baseline DBP and risk of amputation in patients with SGLT2i treatment. d The association between weigh change and risk of PAD in patients with SGLT2i treatment. e The association between weigh change difference and risk of PAD in patients with SGLT2i treatment. $\mathbf{f}$ The association between SBP change and risk of PAD in patients with SGLT2i treatment. $\mathbf{g}$ The association between weigh change and risk of DF in patients with SGLT2i treatment. $\mathbf{h}$ The association between weigh change difference and risk of DF in patients with SGLT2i treatment. i The association between DBP change and risk of DF in patients with SGLT2i treatment. DBP, diastolic blood pressure; DF, diabetic foot; PAD, peripheral arterial disease; SBP, systolic blood pressure; SGLT2i, sodium glucose co-transporter 2 inhibitor

the use of SGLT2i, when compared with GLP-1RA, was associated with an increased risk of lower limb amputation (incidence rate 2.7 vs. 1.1 events per 1000-person years, hazard ratio 2.32 , 95\% CI 1.37 to 3.91 ) [19]. Therefore, it could be assumed that simply weight reduction through energy restrictions might not increase the risk of lower limb amputation. However, the interactions of diuresis and urinary glucose excretion might additively amplify the transient hemodynamic instability, which might be associated with increased risk of amputation and PAD in patients with SGLT2i treatments. But still, apart from GLP-1RA, the associations between other weight-reducing drugs such as orlistat and risk of lower limb complications remains uncertain and requires further investigations.

Blood pressure is another indicator for the hemodynamic status. Our study showed that baseline diastolic blood pressure, greater systolic blood pressure reduction and greater diastolic blood pressure reduction were significantly associated with increased risks of amputation, PAD and DF respectively in SGLT2i users. Blood pressure, as an important vital sign, could also reflect the peripheral perfusion status. Greater blood pressure reduction could be a result of reduced intravascular volume. As is mentioned earlier, the use of thiazide diuretics might increase the risk of lower limb 
Table 2 Meta-regression analysis for the associated factors and risk of amputation events in patients with SGLT2i treatment

\begin{tabular}{|c|c|c|c|c|c|c|c|}
\hline Parameter & $\beta$ & $95 \% \mathrm{Cl}$ & $P$ value & Parameter & $\beta$ & $95 \% \mathrm{Cl}$ & $P$ value \\
\hline \multicolumn{8}{|l|}{ Demographic characteristic } \\
\hline Age (year old) & -0.090 & $-0.224,0.045$ & 0.163 & Sex (male\%) & 0.013 & $-0.023,0.049$ & 0.780 \\
\hline BMI $\left(\mathrm{kg} / \mathrm{m}^{2}\right)$ & 0.097 & $-0.231,0.426$ & 0.506 & Diabetes duration (year) & 0.177 & $-0.036,0.390$ & 0.984 \\
\hline \multicolumn{8}{|l|}{ HbA1c and weight change } \\
\hline HbA1c change (\%) & 0.689 & $-4.454,5.832$ & 0.761 & Weight change $(\mathrm{kg})^{*}$ & -0.461 & $-0.726,-0.197$ & 0.007 \\
\hline HbA1c change difference (\%) & -0.479 & $-6.171,5.213$ & 0.848 & Weight change difference $(\mathrm{kg})^{*}$ & -0.436 & $-0.795,-0.078$ & 0.026 \\
\hline \multicolumn{8}{|l|}{ Hemodynamic indicator } \\
\hline Baseline SBP (mmHg) & 0.005 & $-0.094,0.105$ & 0.902 & Baseline DBP $(\mathrm{mmHg})^{*}$ & -0.528 & $-0.852,-0.205$ & 0.009 \\
\hline SBP change (mmHg) & -0.254 & $-0.545,0.037$ & 0.075 & DBP change (mmHg) & -0.279 & $-0.638,0.079$ & 0.096 \\
\hline Volume depletion (event\%) & -0.372 & $-0.900,0.155$ & 0.121 & - & - & - & - \\
\hline \multicolumn{8}{|l|}{ Renal function profile } \\
\hline Baseline eGFR (mL/min/1.73m²) & 0.058 & $-0.393,0.508$ & 0.352 & eGFR change $\left(\mathrm{mL} / \mathrm{min} / 1.73 \mathrm{~m}^{2}\right)$ & 0.393 & $-1.183,1.969$ & 0.550 \\
\hline \multicolumn{8}{|l|}{ PAD risk factor } \\
\hline Previous PAD (\%) & 0.003 & $-0.296,0.301$ & 0.973 & - & - & - & - \\
\hline
\end{tabular}

${ }^{*} \mathrm{P}<0.05$. BMI body mass index, SGLT2i sodium glucose co-transporter 2 inhibitor, $P A D$ peripheral arterial disease, $S B P$ systolic blood pressure, $D B P$ diastolic blood pressure, $H C T$ hematocrit, eGFR estimated glomerular filtration rate

Table 3 Meta-regression analysis for the associated factors and risk of PAD events in patients with SGLT2i treatment

\begin{tabular}{|c|c|c|c|c|c|c|c|}
\hline Parameter & $\beta$ & $95 \% \mathrm{Cl}$ & $P$ value & Parameter & $\beta$ & $95 \% \mathrm{Cl}$ & $P$ value \\
\hline \multicolumn{8}{|l|}{ Demographic characteristic } \\
\hline \multicolumn{2}{|c|}{ Age (year old $)-0.057$} & $-0.133,0.018$ & 0.133 & Sex (male\%) & -0.003 & $-0.026,0.019$ & 0.780 \\
\hline $\mathrm{BMI}\left(\mathrm{kg} / \mathrm{m}^{2}\right)$ & -0.011 & $-0.071,0.050$ & 0.720 & Diabetes duration (year) & -0.001 & $-0.104,0.102$ & 0.984 \\
\hline \multicolumn{8}{|l|}{$\mathrm{HbA1c}$ and weight change } \\
\hline \multicolumn{2}{|c|}{ HbA1c change (\%) -0.540} & $-1.518,0.437$ & 0.269 & Weight change $(\mathrm{kg})^{*}$ & -0.359 & $-0.545,-0.172$ & $<0.001$ \\
\hline \multicolumn{2}{|c|}{ HbA1c change difference (\%) - 1.126} & $-3.166,0.914$ & 0.269 & Weight change difference $(\mathrm{kg})^{*}$ & -0.264 & $-0.472,-0.056$ & 0.014 \\
\hline \multicolumn{8}{|c|}{ Hemodynamic indicator } \\
\hline \multicolumn{2}{|c|}{ Baseline SBP $(\mathrm{mmHg}) 0.004$} & $-0.061,0.070$ & 0.896 & Baseline DBP (mmHg) & 0.017 & $-0.338,0.372$ & 0.922 \\
\hline \multicolumn{2}{|c|}{ SBP change $(\mathrm{mmHg})^{*}-0.207$} & $-0.390,-0.023$ & 0.028 & DBP change (mmHg) & -0.191 & $-0.493,0.110$ & 0.201 \\
\hline \multicolumn{2}{|c|}{ Hemoglobin change $(\mathrm{g} / \mathrm{L})-0.076$} & $-0.746,0.594$ & 0.782 & HCT change & -0.242 & $-1.117,0.634$ & 0.552 \\
\hline \multicolumn{2}{|c|}{ Volume depletion (event\%) - 0.061} & $-0.269,0.147$ & 0.528 & Osmotic diuresis (event\%) & 0.367 & $-0.091,0.825$ & 0.091 \\
\hline \multicolumn{8}{|l|}{ Renal function profile } \\
\hline Baseline eGFR $\left(\mathrm{mL} / \mathrm{min} / 1.73 \mathrm{~m}^{2}\right)$ & 0.019 & $-0.014,0.051$ & 0.242 & eGFR change (mL/min/1.73m²) & -0.184 & $-0.473,0.107$ & 0.200 \\
\hline \multicolumn{8}{|l|}{ PAD risk factor } \\
\hline \multicolumn{2}{|c|}{ Previous PAD (\%) 0.017} & $-0.039,0.072$ & 0.411 & - & - & - & - \\
\hline
\end{tabular}

${ }^{*} \mathrm{P}<0.05$. BMI body mass index, SGLT2i sodium glucose co-transporter 2 inhibitor, $P A D$ peripheral arterial disease, $S B P$ systolic blood pressure, $D B P$ diastolic blood pressure, $H C T$ hematocrit, eGFR estimated glomerular filtration rate

amputation in patients with T2D [9]. The anti-hypertensive mechanism of thiazide diuretics is to reduce water and sodium retention, which again suggested that the diuresis and blood pressure reduction might be associated with the increased risk of lower limb complications observed in these very patients. However, the evidence regarding other kinds of blood pressure lowering drugs and the risk of lower limb complications is so far limited. Whether the associations of the blood pressure lowering and the risk of lower limb complications that observed in thiazide diuretics may also apply to a broad spectrum of hypotensive agents remains to be evaluated. Moreover, our previous metaanalysis of 94 RCTs showed that systolic blood pressure reduction in patients with SGLT2i was significantly associated with weight reduction [20]. It indicates that continuous weight reduction in the later phase caused by loss of adiposity might also facilitate the blood pressure reduction in patients with SGLT2i treatment, which further interrupts the hemodynamic hemostasis 
Table 4 Meta-regression analysis for the associated factors and risk of DF events in patients with SGLT2i treatment

\begin{tabular}{|c|c|c|c|c|c|c|c|}
\hline Parameter & $\beta$ & $95 \% \mathrm{Cl}$ & $P$ value & Parameter & $\beta$ & $95 \% \mathrm{Cl}$ & $P$ value \\
\hline \multicolumn{8}{|l|}{ Demographic characteristic } \\
\hline Age (year old) & -0.089 & $-0.192,0.014$ & 0.087 & Sex (male\%) & -0.021 & $-0.058,0.016$ & 0.247 \\
\hline $\mathrm{BMI}\left(\mathrm{kg} / \mathrm{m}^{2}\right)$ & -0.001 & $-0.060,0.058$ & 0.920 & Diabetes duration (year) & -0.027 & $-0.187,0.133$ & 0.732 \\
\hline \multicolumn{8}{|l|}{$\mathrm{HbA1c}$ and weight change } \\
\hline HbA1c change (\%) & -1.723 & $-3.749,0.303$ & 0.091 & Weight change $(\mathrm{kg})^{*}$ & -0.476 & $-0.836,-0.116$ & 0.012 \\
\hline HbA1c change difference (\%) & 1.305 & $-1.289,3.900$ & 0.307 & Weight change difference $(\mathrm{kg})^{*}$ & -0.437 & $-0.719,-0.155$ & 0.004 \\
\hline \multicolumn{8}{|l|}{ Hemodynamic indicator } \\
\hline Baseline SBP (mmHg) & 0.023 & $-0.085,0.131$ & 0.660 & Baseline DBP (mmHg) & 0.201 & $-0.364,0.767$ & 0.458 \\
\hline SBP change $(\mathrm{mmHg})$ & -0.264 & $-0.541,-0.013$ & 0.060 & DBP change $(\mathrm{mmHg})^{*}$ & -0.312 & $-0.610,-0.015$ & 0.041 \\
\hline Hemoglobin change (g/L) & -0.902 & $-2.010,0.206$ & 0.087 & HCT change & 0.015 & $-1.164,1.193$ & 0.976 \\
\hline Volume depletion (event\%) & -0.007 & $-0.075,0.061$ & 0.772 & - & - & - & - \\
\hline \multicolumn{8}{|l|}{ Renal function profile } \\
\hline Baseline eGFR (mL/min/1.73m²) & -0.003 & $-0.041,0.035$ & 0.867 & eGFR change $\left(\mathrm{mL} / \mathrm{min} / 1.73 \mathrm{~m}^{2}\right)$ & -0.114 & $-0.755,0.527$ & 0.706 \\
\hline \multicolumn{8}{|l|}{ PAD risk factor } \\
\hline Previous PAD (\%) & -0.007 & $-0.075,0.061$ & 0.772 & - & - & - & - \\
\hline
\end{tabular}

${ }^{*} \mathrm{P}<0.05$. BMI body mass index, SGLT2i sodium glucose co-transporter 2 inhibitor, $P A D$ peripheral arterial disease, $D F$ diabetic foot, $S B P$ systolic blood pressure, $D B P$ diastolic blood pressure, $H C T$ hematocrit, eGFR estimated glomerular filtration rate

in the peripheral circulation and in turn contributes to the increased risk of lower limb complications.

It is noted that SGLT2i may also increase diet and water intakes as compensatory feedbacks in response to glycosuria and diuresis. Compensatory increased sugar intake has been reported in patients with T2D under dapagliflozin treatment [21]. However, although daily sucrose intake significantly increased in patients taking dapagliflozin for 3 months, daily intakes of total calories and the proportions of the three major nutrients were not significantly increased in patients either with or without dapagliflozin treatment [21]. Another prospective study in 26 patients with T2D indicated that dapagliflozin resulted in a significantly reduction in body weight by 12 weeks without inducing prominent hyperphagia [22]. As is mentioned before, the diuresis-related weight reduction may last for 4-8 weeks until a new homeostasis of body fluid is established through compensatory water intakes and other hormonal, metabolic and hemodynamic adaptions [11-13]. But during this time, the potential influence of early circulatory insufficiency may still occur. Actually, the weight-loss and blood-pressure-lowering effects have been observed and validated in multiple RCTs with long-term use of SGLT2i. It seems that the compensatory diet and water intakes might not be adequate to fully counteract the effects on body weight and blood pressure by SGLT2i-induced glycosuria and diuresis. In the sense, the reductions in body weight and blood pressure after SGLT2i treatment are still of clinical significance when it comes to lower limb complications in diabetes.
Based on the above analyses, we proposed that, in patients with high risk of lower limb complications, loss of intravascular volume at the early phase with SGLT2i treatment, reflected as diuresis-mediated weight reduction and blood pressure reduction, may contribute to first ischemic damage for the extremities in these patients. Continuous weight reduction at the later phase with SGLT2i treatment would further facilitate the blood pressure reduction and worsen the insufficient distal perfusion, which might contribute to the gradual development of PAD. Surgical removals might be eventually required in some extremely severe cases.

Furthermore, according to our meta-analysis, typical volume depletion indicators such as hematocrit, hemoglobin, were not associated with the increased risks of amputation and PAD. Such inconsistence might be due to the limited available data for the variables mentioned above. However, in patients with mild to moderate loss of body fluid, we assumed that weight reduction and blood pressure reduction rather than typical volume depletion manifestations, could still be observed. Thus, such subclinical volume depletion in patients at high risk requires attention as well.

Our study showed that the risks of amputation was only slightly increased in patients with canagliflozin treatment, but not in other subtypes of SGLT2i. It was proposed that partial inhibition of SGLT1 of canagliflozin might be associated with this subtype difference in inhibiting selectivity [23]. However, we did not observe increased risk of amputation and PAD in patients with sotagliflozin treatment, a dual inhibitor for SGLT1 and 
SGLT2 [24]. Another explanation may lie in the different amplitude of the body weight and blood pressure reduction among different SGLT2i subtypes. Although the pooled effect of all the SGLT2i subtypes could significantly reduce the body weight and blood pressure in patients with diabetes when compared with placebo treatment, our analysis showed that canagliflozin treatment led to the greatest weight reduction and the greatest BP reduction among all SGLT2i subtypes (Additional file 1: Table S3). It is assumed that the intensive amplitude of body weight and BP reduction caused by canagliflozin is more likely to exceed the autoregulation thresholds in patients at risk of lower limb complications while in patients with other subtypes of SGLT2i treatment, the alterations in hemodynamic status might be smoother and steadier. It might be a possible reason why the risk of amputation and PAD is only mildly increased in canagliflozin strata.

Actually, SGLT2i is a revolutionary antidiabetic agent which develops a novel treatment pattern for patients with diabetes. The clinical benefits from SGLT2i are much more than sorely improved glycemic control. SGLT2i confers more favorable pleiotropic effects on body weight, alanine aminotransferase and eGFR changes, potentially improving patients' cardiometabolic disease risk when achieves hypoglycemic effects comparable to antidiabetic agents like dipeptidyl peptidase-4 inhibitor (DPP-4i) [25]. Moreover, studies showed that cardiovascular and renal benefits from SGLT2i treatment might be in part mediated by a state of fasting mimicry via several molecular mechanisms [26] and the regulation of autonomic nervous system [27]. SGLT2i may also ameliorate lipotoxic damage in stearate-treated myeloid angiogenic cells involved in atherosclerotic plaque vulnerability and/or thrombosis [28]. Together with the encouraging outcomes from CVOTs, SGLT2i is recommended as the first-line treatment for patients with arteriosclerotic cardiovascular disease, heart failure and chronic kidney disease in the latest American Diabetes Association Standards of Medical Care in Diabetes-2021, if their eGFRs meet the requirements [29].

In fact, SGLT2i is generally well tolerated in patients with diabetes. But the enhanced precaution action may further reduce the potential risk during the SGLT2i treatment course for patients susceptible to lower limb complications. Our study indicates that the amplitudes of reductions in body weight and blood pressure are associated with risks of lower limb complications, which calls for an additional attention to the alterations in body weight and blood pressure, two common indicators easy and feasible to monitor during the SGLT2i treatment. In studies in which canagliflozin was not included, SGLT2i were associated with lower risks of chronic heart failure and adverse lower limb events compared with DPP4i among patients with T2D and PAD in real-world practice [30]. It might be assumed that smooth and steady reductions in body weight and blood pressure, which do not exceed the autoregulation threshold, may not increase but reduce the risk of lower limb complications instead. In this situation, the hemostasis remains intact and meanwhile reductions in body weight and blood pressure will improve the insulin resistance, relieve the chronic inflammation and protect against vascular endothelial injuries. Therefore, monitoring the reductions in body weight and blood pressure will allow clinicians to adjust the SGLT2i regimens accordingly, which ensures the patients benefit from the SGLT2i treatment to the best extent without developing lower limb complications. The precaution action regarding body weight and blood pressure monitoring will promote a safe application of SGLT2i in the clinical settings.

There were some limitations in our studies. First, although there were adjudication committees for cardiovascular and renal endpoints in CVOTs and ROTs, and for important adverse events like urinary tract infection in efficacy and safety evaluation trials, there was no adjudication committees specially designed and set up for relevant lower limb complications in these RCTs. But as large event-driven RCTs, CVOTs and ROTs were basically with proper study design and reliable data assessments. The consistent results from CVOT and ROT strata enhance the creditability of our primary conclusion. Second, the definitions of PAD vary from trial to trial. To minimize the bias, we have unified the inclusion criteria of pre-specific PAD events and strictly stick to it during the data extraction. Moreover, several important data were not commonly reported in the published trials, including hematocrit, smoking status and previous PAD or amputation history. Actually, we did collect the available data about these alternative hemodynamic indicators and independent risk factors, and conducted the corresponding analyses. However, due to the limited data, we did not observe any significant association. Further analyses are still needed to validate and improve the hemodynamic hypothesis in the future.

\section{Conclusions}

According to our meta-analysis, compared with nonSGLT2i users, the risks of amputation and PAD were slightly increased in patients with canagliflozin treatment. Greater reductions in body weight and blood pressure might be associated with the increased risk of lower limb complications in SGLT2i users. Monitoring body weight and blood pressure reduction might be an important risk precaution action for patients at high risk of 
lower limb complications during the SGLT2i treatment course. It might help the health care professionals achieve a safe application of SGLT2i in the clinical settings.

\begin{abstract}
Abbreviations
Cl: Confidence interval; CVOT: Cardiovascular outcome trial; DF: Diabetic foot; DPP-4i: Dipeptidyl peptidase-4 inhibitor; eGFR: Estimated glomerular filtration rate; FDA: Food and Drug Administration; GLP-1RA: Glucagon-like peptide-1 receptor analog; OR: Odd ratio; PAD: Peripheral arterial disease; RCT: Randomized controlled trial; ROT: Renal outcome trial; SGLT2i: Sodium glucose co-transporter 2 inhibitor;T1D: Type 1 diabetes;T2D:Type 2 diabetes; WMD: Weighted mean difference.
\end{abstract}

\section{Supplementary Information}

The online version contains supplementary material available at https://doi. org/10.1186/s12933-021-01276-9.

Additional file 1: Figure S1. Flowchart of included randomized controlled trials of SGLT2i treatment. Figure S2. The funnel plot of included randomized controlled trials of SGLT2i treatment in amputation analysis. Figure S3. The funnel plot of included randomized controlled trials of SGLT2i treatment in PAD analysis. Figure S4. The funnel plot of included randomized controlled trials of SGLT2i treatment in DF analysis. Table S1. The baseline characteristics of included trials of SGLT2i treatment. Table S2. The risk of bias for included trials of SGLT2i treatment. Table S3. Body weight and blood pressure reductions in patients with SGLT2i treatment.

\section{Acknowledgements}

We thank the doctors, nurses and technicians for their practical work during the study at Department of Endocrinology and Metabolism in Peking University People's Hospital.

\section{Authors' contributions}

$\amalg$ and $X C$ conceptualized this study and designed the systematic review protocol; $\mathrm{CL}, \mathrm{XZ}, \mathrm{NL}$, WY and FL performed the study selection and data extraction; $\mathrm{CL}$ and $\mathrm{XC}$ performed the statistical analyses; $\mathrm{CL}, \mathrm{XZ}$ and $\mathrm{XC}$ prepared the outlines and wrote the manuscript. All authors contributed to the critical revision of manuscript drafts.

\section{Funding}

This work was supported by Beijing Natural Science Foundation (No. 7202216) and National Natural Science Foundation of China (Nos. 81970698 and 81970708). The funding agencies had no roles in the study design, data collection or analysis, decision to publish or preparation of the manuscript.

\section{Availability of data and materials}

All data relevant to the study are included in the article or uploaded as additional file. No more additional data are available.

\section{Declarations}

Ethical approval and consent to participate Not applicable.

\section{Consent for publicaton}

Not applicable.

\section{Competing interests}

$L J$ has received fees for lecture presentations and for consulting from AstraZeneca, Merck, Metabasis, MSD, Novartis, Eli Lilly, Roche, Sanofi-Aventis and Takeda. All authors have completed the ICMJE uniform disclosure form at www.icmje.org/coi_disclosure.pdf (available on request from the corresponding author) and declare. No other support from any organization for the submitted work other than that described above.

\section{Author details}

'Department of Endocrinology and Metabolism, Peking University People's Hospital, Beijing, China. ${ }^{2}$ Department of Endocrinology and Metabolism, Beijing Airport Hospital, Beijing, China.

Received: 11 January 2021 Accepted: 15 April 2021

Published online: 28 April 2021

\section{References}

1. Kluger AY, Tecson KM, Lee AY, Lerma EV, Rangaswami J, Lepor NE, Cobble ME, McCullough PA. Class effects of SGLT2 inhibitors on cardiorenal outcomes. Cardiovasc Diabetol. 2019;18(1):99.

2. Neal B, Perkovic V, Mahaffey KW, de Zeeuw D, Fulcher G, Erondu N, Shaw W, Law G, Desai M, Matthews DR, CANVAS Program Collaborative Group. Canagliflozin and cardiovascular and renal events in type 2 diabetes. N Engl J Med. 2017:377(7):644-57.

3. Scheen AJ. Does lower limb amputation concern all SGLT2 inhibitors? Nat Rev Endocrinol. 2018:14:326-28.

4. https://www.fda.gov/drugs/drug-safety-and-availability/fda-drug-safetycommunication-fda-confirms-increased-risk-leg-and-foot-amputationsdiabetes-medicine.

5. Miyashita S, Kuno T, Takagi H, Sugiyama T, Ando T, Valentin N, Shimada YJ, Kodaira M, Numasawa Y, Kanei Y, Bangalore S. Risk of amputation associated with sodium-glucose co-transporter 2 inhibitors: a metaanalysis of five randomized controlled trials. Diabetes Res Clin Pract. 2020;163:108136.

6. Heyward J, Mansour O, Olson L, Singh S, Alexander GC. Association between sodium-glucose cotransporter 2 (SGLT2) inhibitors and lower extremity amputation: a systematic review and meta-analysis. PLoS ONE. 2020;15(6):e0234065.

7. Fralick M, Kim SC, Schneeweiss S, Everett BM, Glynn RJ, Patorno E. Risk of amputation with canagliflozin across categories of age and cardiovascular risk in three US nationwide databases: cohort study. BMJ. 2020;370:m2812.

8. Marso SP, Hiatt WR. Peripheral arterial disease in patients with diabetes. J Am Coll Cardiol. 2006:47(5):921-9.

9. Erkens JA, Klungel OH, Stolk RP, Spoelstra JA, Grobbee DE, Leufkens HG. Antihypertensive Drug Therapy and the Risk of Lower Extremity Amputations in Pharmacologically Treated Type 2 Diabetes Patients. Pharmaco epidemiol Drug Saf. 2004;13(3):139-46.

10. Tanaka A, Node K. Increased amputation risk with canagliflozin treatment: behind the large cardiovascular benefit? Cardiovasc Diabetol. 2017; 16(1):129.

11. Hirose S, Nakajima S, Iwahashi Y, Seo A, Takahashi T, Tamori Y. Impact of the 8-week Administration of tofogliflozin for glycemic control and body composition in Japanese patients with type 2 diabetes mellitus. Intern Med. 2016;55(22):3239-45.

12. Kawata T, lizuka T, lemitsu K, Takihata M, Takai M, Nakajima S, Minami N, Umezawa S, Kanamori A, Takeda H, Ito S, Kikuchi T, Amemiya H, Kaneshiro M, Mokubo A, Takuma T, Machimura H, Tanaka K, Asakura T, Kubota A, Aoyanagi S, Hoshino K, Ishikawa M, Matsuzawa Y, Obana M, Sasai N, Kaneshige H, Minagawa F, Saito T, Shinoda K, Miyakawa M, Tanaka Y, Terauchi Y, Matsuba I. Ipragliflozin improves glycemic control and decreases body fat in patients with type 2 diabetes mellitus. J Clin Med Res. 2017:9(7):586-95.

13. Leiter LA, Yoon KH, Arias P, Langslet G, Xie J, Balis DA, Millington D, Vercruysse F, Canovatchel W, Meininger G. Canagliflozin provides durable glycemic improvements and body weight reduction over 104 weeks versus glimepiride in patients with type 2 diabetes on metformin: a randomized, double-blind, phase 3 study. Diabetes Care. 2015;38(3):355-64.

14. Ferrannini E, Muscelli E, Frascerra S, Baldi S, Mari A, Heise T, Broedl UC, Woerle HJ. Metabolic response to sodium-glucose cotransporter 2 inhibition in type 2 diabetic patients. J Clin Invest. 2014;124(2):499-508.

15. Meloni AR, DeYoung MB, Lowe C, Parkes DG. GLP-1 receptor activated insulin secretion from pancreatic $\beta$-cells: mechanism and glucose dependence. Diabetes Obes Metab. 2013;15(1):15-27.

16. Campbell JE, Drucker DJ. Pharmacology, physiology and mechanisms of incretin hormone action. Cell Metab. 2013;17(6):819-37. 
17. Cai X, Ji L, Chen Y, Yang W, Zhou L, Han X, Zhang S, Ji L. Comparisons of weight changes between sodium-glucose cotransporter 2 inhibitors treatment and glucagon-like peptide-1 analogs treatment in type 2 diabetes patients: a meta-analysis. J Diabetes Investig. 2017;8(4):510-7.

18. Patorno E, Pawar A, Bessette LG, Kim DH, Dave C, Glynn RJ, Munshi MN, Schneeweiss S, Wexler DJ, Kim SC. Comparative effectiveness and safety of sodium-glucose cotransporter 2 inhibitors versus glucagon-like peptide 1 receptor agonists in older adults. Diabetes Care. 2021;44(3):826-35.

19. Ueda $P$, Svanström $H$, Melbye $M$, Eliasson $B$, Svensson AM, Franzén $S$, Gudbjörnsdottir S, Hveem K, Jonasson C, Pasternak B. Sodium glucose cotransporter 2 inhibitors and risk of serious adverse events: nationwide register based cohort study. BMJ. 2018;363:k4365.

20. Hu M, Cai X, Yang W, Zhang S, Nie L, Ji L. Effect of hemoglobin A1c reduction or weight reduction on blood pressure in glucagon-like peptide-1 receptor agonist and sodium-glucose cotransporter-2 inhibitor treatment in type 2 diabetes mellitus: a meta-analysis. J Am Heart Assoc. 2020;9(7):e015323.

21. Horie I, Abiru N, Hongo R, Nakamura T, Ito A, Haraguchi A, Natsuda S, Sagara I, Ando T, Kawakami A. Increased sugar intake as a form of compensatory hyperphagia in patients with type 2 diabetes under dapagliflozin treatment. Diabetes Res Clin Pract. 2018;135:178-84.

22. Kosugi R, Nakatani E, Okamoto K, Aoshima S, Arai H, Inoue T. Effects of sodium-glucose cotransporter 2 inhibitor (dapagliflozin) on food intake and plasma fibroblast growth factor 21 levels in type 2 diabetes patients. Endocr J. 2019;66(8):677-82.

23. Ohgaki R, Wei L, Yamada K, Hara T, Kuriyama C, Okuda S, Ueta K, Shiotani M, Nagamori S, Kanai Y. Interaction of the sodium/glucose cotransporter (SGLT) 2 inhibitor canagliflozin with SGLT1 and SGLT2: inhibition kinetics, sidedness of action, and transporter-associated incorporation accounting for its pharmacodynamic and pharmacokinetic features. J Pharmacol Exp Ther. 2016;358(1):94-102.
24. Sims H, Smith KH, Bramlage P, Minguet J, Sotagliflozin. A dual sodiumglucose co-transporter-1 and -2 inhibitor for the management of type 1 and type 2 diabetes mellitus. Diabet Med. 2018;35(8):1037-48.

25. Shao SC, Chang KC, Lin SJ, Chien RN, Hung MJ, Chan YY, Kao Yang YH, Lai EC. Favorable pleiotropic effects of sodium glucose cotransporter 2 inhibitors: head-to-head comparisons with dipeptidyl peptidase-4 inhibitors in type 2 diabetes patients. Cardiovasc Diabetol. 2020;19(1):17.

26. Packer M. SGLT2 inhibitors produce cardiorenal benefits by promoting adaptive cellular reprogramming to induce a state of fasting mimicry: a paradigm shift in understanding their mechanism of action. Diabetes Care. 2020;43(3):508-11.

27. Spallone V, Valensi P. SGLT2 inhibitors and the autonomic nervous system in diabetes: a promising challenge to better understand multiple target improvement. Diabetes Metab. 2021;47(4):101224.

28. Spigoni V, Fantuzzi F, Carubbi C, Pozzi G, Masselli E, Gobbi G, Solini A, Bonadonna RC, Dei Cas A. Sodium-glucose cotransporter 2 inhibitors antagonize lipotoxicity in human myeloid angiogenic cells and ADPdependent activation in human platelets: potential relevance to prevention of cardiovascular events. Cardiovasc Diabetol. 2020;19(1):46.

29. American Diabetes Association. Pharmacologic approaches to glycemic treatment: standards of medical care in diabetes-2021. Diabetes Care. 2021;44(S1):111-24.

30. Lee HF, Chen SW, Liu JR, Li PR, Wu LS, Chang SH, Yeh YH, Kuo CT, Chan YH, See LC. Major adverse cardiovascular and limb events in patients with diabetes and concomitant peripheral artery disease treated with sodium glucose cotransporter 2 inhibitor versus dipeptidyl peptidase-4 inhibitor. Cardiovasc Diabetol. 2020;19(1):160.

\section{Publisher's note}

Springer Nature remains neutral with regard to jurisdictional claims in published maps and institutional affiliations.
Ready to submit your research? Choose BMC and benefit from:

- fast, convenient online submission

- thorough peer review by experienced researchers in your field

- rapid publication on acceptance

- support for research data, including large and complex data types

- gold Open Access which fosters wider collaboration and increased citations

- maximum visibility for your research: over $100 \mathrm{M}$ website views per year

At BMC, research is always in progress.

Learn more biomedcentral.com/submissions 